\title{
Special edition update on postmenopausal osteoporosis
}

Dale W. Stovall

Published online: 6 January 2011

(C) Springer Science+Business Media, LLC 2010

\section{Introduction}

For the patient with postmenopausal osteoporosis, the clinician who evaluates and treats her, and the investigator who works to clarify controversial issues and identify safe and efficacious new treatments for this disease, the first decade of this new century has been an exciting one. The medical literature regarding osteoporosis is growing rapidly. A new and dynamic tool to assess the absolute risk for fracture with a global perspective, FRAX, is now available. Several therapeutic agents already on the market have now been evaluated for long-term safety and efficacy. Some agents have become available in a generic form. A few new medical therapies have come to the marketplace, and other treatment modalities are currently under investigation.
However, even as we learn more in regards to the evaluation and treatment of this disease, many questions still remain and some new questions have arisen. These questions include when to initiate an assessment for the disease, who is the best candidate to receive a pharmaceutical agent, which agent should be selected for a given patient, how do we develop better treatment options, how do we improve persistence with therapy, is long-term therapy with a potent agent safe, and how to follow patients on treatment. This Special Edition on postmenopausal osteoporosis addresses many of these important clinical questions in an effort to update the patient, clinician, and investigator on what we currently know about how to assess, treat, and follow patients with osteoporosis.

D. W. Stovall $(\bowtie)$

Departments of Obstetrics and Gynecology and Internal Medicine,

Division of Reproductive Endocrinology,

UVA Northridge Medical Complex, Suite 104, 2955 Ivy Road,

Charlottesville, VA 22903, USA

e-mail: ds2as@virginia.edu 